\section{Cahiers de Narratologie}

Analyse et théorie narratives

17 | 2009

Stéréotype et narration littéraire

\title{
«C'est alors que le Corsaire Sanglot... ». Le stéréotype romanesque dans les romans surréalistes des années vingt
}

Ivanne Rialland

\section{CpenEdition}

Journals

Édition électronique

URL : http://journals.openedition.org/narratologie/1199

DOI : 10.4000/narratologie.1199

ISSN : 1765-307X

Éditeur

LIRCES

\section{Référence électronique}

Ivanne Rialland, " "C'est alors que le Corsaire Sanglot... ». Le stéréotype romanesque dans les romans surréalistes des années vingt », Cahiers de Narratologie [En ligne], 17 | 2009, mis en ligne le 04 janvier 2010, consulté le 14 novembre 2019. URL : http://journals.openedition.org/narratologie/1199 ; DOI : 10.4000/narratologie.1199

Ce document a été généré automatiquement le 14 novembre 2019.

\section{cc) (i) (2)}

Cahiers de Narratologie - Analyse et théorie narratives est mis à disposition selon les termes de la licence Creative Commons Attribution - Pas d'Utilisation Commerciale - Pas de Modification 4.0 International. 


\title{
«C'est alors que le Corsaire Sanglot... ». Le stéréotype romanesque dans les romans surréalistes des années vingt
}

\author{
Ivanne Rialland
}

1 En dépit de l'anathème jeté par Breton sur le roman dans le Manifeste du surréalisme en 1924, les surréalistes ont écrit des romans, et ce dès les années vingt, comme l'a montré la thèse de Jacqueline Chénieux-Gendron ${ }^{1}$. Ce constat a permis une analyse plus fine des propos de Breton : ce que condamne Breton, ce n'est pas tant le roman que son avatar réaliste et psychologique qui domine la production romanesque des années vingt ${ }^{2}$, et, à travers lui, les préjugés sur lesquels repose l'attitude réaliste. La critique des stéréotypes romanesques dans la description et l'étude des caractères est indissociable de la critique de cette attitude qui plaque, comme le prétendu roman réaliste, ses stéréotypes sur le réel: "l'intraitable manie qui consiste à ramener l'inconnu au connu $^{3}$ » règne autant dans l'écriture que dans la vie. Et c'est également du côté du roman - du roman noir - que Breton va trouver le merveilleux permettant de renouveler la vision du réel ${ }^{4}$.

2 Le surréalisme va ainsi puiser dans toute une littérature romanesque populaire un imaginaire insolite et merveilleux qui nourrit autant l'écriture poétique que narrative. Cependant, si les fragments de cette littérature populaire, insérés dans un poème ou un texte automatique, changent de sens et de portée, les « grosses ficelles » de la série des Fantômas, pour citer l'exemple le plus célèbre, posent la question de leur utilisation dans la narration surréaliste. L'accentuation du suspense peut, d'une part, aller de pair avec la recherche surréaliste de la surprise, mais la banalité de cette littérature semble, d'autre part, contredire la quête du nouveau et de l'accès au réel véritable qu'est le surréel.

3 C'est cette articulation entre la poétique surréaliste et le stéréotype romanesque, jouant au niveau du personnage, de la scène et du ressort narratif, que nous aimerions 
analyser ici, en nous concentrant sur un corpus de romans des années vingt où la question est posée avec une particulière acuité : Anicet ou le panorama, roman (1921) d'Aragon, À la dérive (1923) et Les Dernières nuits de Paris (1928) de Soupault, La liberté ou l'amour! (1927) de Desnos, Babylone (1927) et Êtes-vous fous? (1929) de Crevel. L'usage du stéréotype y est, à nos yeux, de quatre ordres, narratologique, critique, érotique et existentiel, liés en vue d'une déconstruction, du réel comme du roman, qui n'est pas dissociable d'une jouissance de l'écriture et de la lecture romanesques.

Pour écrire de (faux) romans

4 Comme le souligne Marie-Claire Dumas ${ }^{5}$, l'expérience de l'automatisme a révélé aux surréalistes l'imprégnation du spontané par le déjà-dit. Les textes de Soupault des Champs magnétiques portent la trace de réminiscences littéraires qui structurent en sous-main le flux de l'écriture, comme en témoigne «En 80 jours $^{6}$ ». C'est ce rôle structurant et générateur que semble d'abord avoir le stéréotype romanesque dans le roman surréaliste : les scènes convenues - l'enlèvement, l'aveu - les " grosses ficelles » - coïncidences, reconnaissances - lancent ou relancent la narration en fonctionnant à ce niveau comme un « secret de l'art magique surréaliste »:

Si le silence menace de s'établir [...], rompez sans hésiter avec une ligne trop claire.

À la suite du mot dont l'origine vous semble suspecte, posez une lettre quelconque,

la lette $l$ par exemple, toujours la lettre $l$, et ramenez l'arbitraire en imposant cette

lette pour initiale au mot qui suivra?.

Le titre du chapitre où se produit la rencontre de Mirabelle et d'Anicet, «L'aventure de la chambre ", annonce ainsi une scène romanesque-type, où le partage d'une chambre d'auberge par deux voyageurs entraîne une aventure, parfois galante. La scène se déroule en effet "suivant les rites $^{8}$ ", comme le souligne l'auteur. D'ailleurs le personnage principal en a tout à fait conscience, Anicet calquant son comportement sur celui qui est prévu par le stéréotype. Il déclare par exemple aux personnages masqués qui ont fait irruption dans la chambre de Mirabelle: «[...] il est d'habitude immémoriale qu'en pareille circonstance le personnage au visage nu objurgue ses compagnons masqués de quitter leur anonymat, et reconnaissez que ce serait méconnaître toutes les traditions de ne pas accéder à cette demande9. "

C'est le stéréotype romanesque qui noue également l'intrigue des Dernières Nuits de Paris, avec une réversion de l'interprétation faite par le narrateur sur la diégèse. Celuici, en effet, analyse des faits épars - une scène étrange devant l'Institut, la rencontre d'un marin - en y appliquant un schéma romanesque qui lui est suggéré par le journal du lendemain: la femme qu'il a vue devant l'Institut est la compagne du marin rencontré, qui n'est autre que l'assassin qu'évoque le journal. L'hypothèse la plus romanesque, dans ce roman, étant toujours la meilleure, la suite de l'histoire confirme son exactitude et multiplie reconnaissances et coïncidences qui organisent autour des figure de Volpe et de Georgette l'ensemble des faits narrés. D'une manière similaire, la prédiction de la voyante au début d'Êtes-vous fous? propose un cadre romanesque stéréotypé au texte qui s'ébauche: «D'abord un mariage avec une rousse. Vous aurez été présenté à la fiancée, à l'étranger, au cours d'un voyage ${ }^{10}$. 》 Celui-ci sera en partie démenti, puisque Vagualame ne fera pas l'enfant bleu prédit avec la rouquine qui se révèlera lesbienne ${ }^{11}$. Mais, de pair avec le résumé liminaire des chapitres qui fait signe du côté du roman-feuilleton, ce programme romanesque assure la dimension narrative $\mathrm{du}$ texte en l'orientant vers un futur et en provoquant les capacités d'anticipation du lecteur ${ }^{12}$. 
7 Le stéréotype paraît donc assurer un rôle d'entrée dans le régime romanesque, comme le montre tout particulièrement La liberté ou l'amour ! :

Le vent apportait des feuilles arrachées aux arbres des Tuileries et ces feuilles tombaient avec un bruit mou. C'étaient des gants; gants de toutes sortes, gants de peau, gants de Suède, gants de fil longs. C'est devant le bijoutier une femme qui se dégante pour essayer une bague et se faire baiser la main par le Corsaire Sanglot, c'est une chanteuse, au fond d'un théâtre houleux, venant avec des effluves de guillotine et des cris de Révolution, c'est le peu d'une main qu'on peut voir au niveau des boutons. [...]

Revenu sur mes pas et longeant les arcades de la rue de Rivoli, je vis enfin Louise Lame marcher devant moi $^{13}$.

Le roman surgit d'un passage de la figure à la fiction, provoqué par une association d'idées déterminée par le stéréotype. Le personnage de Louise Lame nait de la vision des feuilles qui tombent, reliées, par le biais de l'analogie de forme entre feuilles et gants, au stéréotype de la femme fatale, renforcé par l'idée de mort qu'amène l'analogie des feuilles mortes et des têtes coupées. Le narrateur suit alors Louise Lame qui se met à s'effeuiller, le texte continuant à être construit par analogie. Les pièces de vêtements abandonnées suscitent à leur tour des micro-récits venus tout droit des romans d'aventures aimés de Desnos : l'odeur d'ambre du pantalon évoque des pêcheurs sur la banquise, le manteau de fourrure un léopard dans la jungle. Ce dernier récit dérive vers le fantastique, puisqu'on assiste au strip-tease du léopard qui apporte sa peau en hommage à « la fatale et adorable fille ${ }^{14}$ ». Les récits puisés dans un arsenal romanesque type tendent ainsi à rejoindre et à nourrir le récit-cadre qu'ils relancent. Le feuilletage paradigmatique est projeté sur l'axe narratif pour générer un récit par épisodes caractéristique de la littérature romanesque, et dont la dernière phrase est emblématique : « C'est alors que le Corsaire Sanglot... » L'aposiopèse, en laissant ouvert le roman, désigne le rôle d'entraînement narratif que joue le stéréotype romanesque, qui se situe en réalité à un niveau de généralité plus grand que la simple scène-type : ce sont les procédés de retard et de réserve générateurs de suspense et de curiosité qu'empruntent ces romans surréalistes, soit les ficelles qui font exclure le roman populaire de la littérature sérieuse.

\section{Le stéréotype contre le roman}

En empruntant ces ressorts usés de la littérature romanesque et en les désignant, les surréalistes invitent à une lecture ludique de leurs romans. Aragon multiplie, dans Anicet ou le panorama, roman, les interventions d'auteur, soulignant, et ce dès le titre, la panoplie de stéréotypes auxquels il recourt et dont Anicet, on l'a vu, a conscience. Un bon exemple de cette utilisation ludique est la présence dans le roman de Nick Carter, dont les différents déguisements permettent de répéter, de façon gratuite, les reconnaissances générant la surprise :

À peine était-il sorti que l'expression niaise s'effaça sur le visage du Bolonais; les yeux du critique lançaient des flammes et le lecteur devina sans peine en lui l'Américain du Café Biard que, par un trait de génie, il assimila sur l'heure au grand détective Nick Carter dont la venue dans cette histoire n'avait été pourtant qu'occasionnellement pressentie ${ }^{15}$.

Dans Anicet, la dimension ludique du stéréotype est doublée par les références intertextuelles. Le déguisement d'Omme au moment de la tentative d'enlèvement de Mirabelle fait surgir la silhouette de Fantômas : «D'en bas, Anicet et Mirabelle y virent se dresser, la main gauche sur le battant ouvert, un grand personnage masqué de velours, coiffé d'un haut-de-forme et drapé dans une cape à $\operatorname{collet}^{16}[. .$.$] .» L'effet$ 
dramatique est immédiatement brisé par Mirabelle qui se moque de cette «mascarade ${ }^{17}$ ", qui permet ensuite, autre stéréotype romanesque, de susciter un quiproquo, Anicet prenant la place d'Omme auprès de ses complices.

Si Aragon s'amuse des ficelles du roman populaire, en même temps, elles garantissent la progression de l'action en évitant sa motivation par la psychologie du personnage que critique Breton dans le Manifeste :

L'auteur s'en prend à un caractère, et, celui-ci étant donné, fait pérégriner son héros à travers le monde. Quoi qu'il arrive, ce héros, dont les actions et les réactions sont admirablement prévues, se doit de ne pas déjouer, tout en ayant l'air de les déjouer, les calculs dont il est l'objet. Les vagues de la vie peuvent paraître l'enlever, le rouler, le faire descendre, il relèvera toujours de ce type humain formé $e^{18}$.

Les actions qui s'enchaînent ne sont pas, dans Anicet, déterminées par le caractère du héros, par une stéréotypie psychologique, mais par une stéréotypie romanesque qui évacue la question psychologique. Si Anicet, embrigadé dans une bande de malfaiteurs, se trouve à cambrioler l'atelier de Bleu, ce n'est pas en raison de la rivalité amoureuse qui les oppose, mais parce que cette coïncidence est appelée par l'horizon d'attente romanesque : «"Je suis chez Bleu." Il y avait un siècle que tout le monde le savait ${ }^{19}$. » De même, l'appariement des personnages à des types romanesques bien connus rend sans pertinence le recours à une quelconque motivation psychologique : le Corsaire Sanglot est un aventurier, Louise Lame dans La liberté ou l'amour!, Yolande dans Êtes-vous fous? et Cynthia dans Babylone des femmes fatales, le marin des Dernières nuits de Paris un assassin.

L'emprunt au romanesque populaire de séquences stéréotypées tend, en outre, à découper dans la narration des scènes qui sont des pics romanesques: la pêcheuse de perles dans La liberté ou l'amour ! est introduite au moment même de son suicide qu'elle offre à son amant ${ }^{20}$, le détective rencontré par David Aubry dans le train livre immédiatement, comme un morceau choisi de detective novel, la description de la scène de crime de «la fameuse histoire de l'hôtel Californy ${ }^{21}$ ». Il se produit une densification de la narration qui fait l'économie des "moments nuls ${ }^{22}$ » que dénonce Breton. En deux pages à peine, Vagualame rencontre Yolande, la femme fatale, qui se présente explicitement comme telle et l'emmène aussitôt chez elle : «Je suis Yolande, la belle Yolande, femme fatale. Soyons $\mathrm{amis}^{23}$. » En cela, ces romans surréalistes accomplissent le type du roman d'aventures tel que le décrit Rivière par opposition au roman symboliste :

Ici nous avons un créateur qui marche parmi ses inventions, comme un voyageur entre des taillis ; il n'y voit pas à plus de quatre pas [...]. Il est en face de son œuvre, comme il est en face du monde : là où il se trouve en elle, c'est toujours le plus loin qu'il soit allé ; tout le reste est encore de l'avenir pour lui. C'est qu'il ne s'entend guère à percer le voile des idées. Tous ses dons sont de création, au lieu d'être de perspicacité ; il dépense tout son génie à soulever son histoire et il n'en a plus pour se détacher d'elle et aller à l'avance jusqu'au bout ${ }^{24}[. .$.$] .$

L'accumulation de péripéties et de reconnaissances et l'absurdité que crée la rencontre dans un même espace textuel du detective novel, du roman de pionniers et du roman de pirates, pour donner l'exemple d'À la dérive, provoquent cet aveuglement, cette imprédictibilité de la suite narrative qui est d'autant plus sensible que la présence de morceaux de romans intrigués favorise l'activité anticipatrice du lecteur qui s'attend à ce que la tension suscitée par le texte soit dénouée ${ }^{25}$. 
15 Si la vraisemblance du roman réaliste et psychologique est mise à mal par le stéréotype romanesque, celui-ci est également dénoncé dans sa banalité, et Crevel se livre à une parodie réjouissante des romans sentimentaux et édifiants sous le tire de cour de goitreuse :

Goitreuse, par esprit fédéral de renoncement elle a sacrifié la part quasi divine d'elle-même, et c'est de son histoire, toute son histoire, rien que son histoire, que s'est inspirée la réplique helvète à Cœur de Française, car enfin, Vagualame l'a deviné, ne font qu'une seule et même personne cette Schwester et l'héroïne de Couur de goitreuse, la grande œuvre du célèbre romancier vaudois qui exalte avec un juste lyrisme la costaude, pataude, rougeaude, rustaude, suissaude vertu des montagnards ${ }^{26}[\ldots] \ldots$

16 De même, dans Les Dernières Nuits de Paris, Soupault dénonce le caractère fabriqué du mystère recherché par Volpe et sa bande :

Ce qui frappait en effet chez presque tous ces gens c'était leur goût invraisemblable de l'extraordinaire. Ils avaient besoin de se croire en butte aux dangers les plus extravagants. [...] Volpe lui-même avait besoin du fantastique. [...] Ils avaient tous pris l'habitude d'inventer, de tromper, de préparer des fausses pistes, des alibis de toute sorte $e^{27}$.

17 Cependant, le stéréotype romanesque fait généralement l'objet d'une mise à distance ludique, qui rompt, certes, l'illusion référentielle visée par le roman populaire, mais qui est avant tout dirigée contre la cohérence et la logique qui président à l'écriture réaliste autant qu'à l'attitude réaliste dénoncée par Breton.

Une érotique du stéréotype

$18 \mathrm{Si}$, bien sûr, les surréalistes ne font pas un usage naïf des stéréotypes romanesques, il faut d'abord souligner avec Paul Bleton ${ }^{28}$ que la lecture ludique peut tout à fait être programmée par la paralittérature, qui suppose une forte compétence générique et thématique et abonde de ce fait en formes parodiques. Ensuite, cette utilisation n'est pas simplement déconstructrice : il y a un plaisir du stéréotype, dont témoigne le goût qu'ont les surréalistes pour la littérature populaire. Philippe Soupault aurait ainsi écrit La Mort de Nick Carter pour se débarrasser de la fascination qu'il éprouvait pour le détective. Le stéréotype romanesque intervient dans le roman surréaliste comme la trace d'un plaisir de lecture, qui est aussi un plaisir de répétition, lié à celui du fantasme érotique dans La liberté ou l'amour ! Marie-Paule Berranger montre en effet que La liberté ou l'amour! «tente de mettre à distance l'obsession amoureuse par une écriture elle-même obsessionnelle ", Desnos jouant la "passion du récit » contre la "passion amoureuse ${ }^{29}$ ». Desnos utilise également contre cette dernière le fantasme érotique, qui prend peu à peu corps dans le récit pour s'incarner dans la scène de flagellation $\mathrm{du}$ pensionnat d'Humming-Bird Garden, alors que l'émergence du sentiment amoureux arrête la narration: «Qu'elle vienne, celle que j'aimerai, au lieu de vous raconter des histoires merveilleuses (j'allais dire à dormir debout ${ }^{30}$ ). » Stéréotype romanesque et fantasme se rejoignent par leur dimension narrative et par la pulsion de répétition qui les gouverne, au point qu'une scène de roman de l'Ouest Desnos connaissait presque par cœur les œuvres de Gustave Aimard - se substitue à la scène de la fellation de Corsaire Sanglot :

Quand la respiration de Corsaire Sanglot se fit haletante, Louise Lame devint plus resplendissante que le mâle.

[...]

Et la pensée de Corsaire Sanglot suivait une piste au cœur de la forêt vierge. 
Il arriva dans une ville de chercheurs d'or. Dans un bal dansait une Espagnole vêtue de façon excitante ${ }^{31}$.

Survient une scène de combat qui provoque la fuite de l'Espagnole et de Corsaire Sanglot. Celui-ci arrive alors à Paris, sauve une femme d'une maison en flammes nouveau stéréotype romanesque; cette scène du sauvetage conduit de façon impromptue à la première évocation du pensionnat anglais qui est le théâtre du fantasme principal du roman, évocation interrompue par des points de suspension marquant la jouissance de Corsaire Sanglot, indiquée, en creux, par la réplique de Louise Lame qui suit immédiatement: «Dis-moi que tu m'aimes! râla Louise Lame éperdue ${ }^{32}$. »

On peut analyser dans le même registre érotique l'aposiopèse finale. Comme le montre très bien Raphaël Baroni ${ }^{33}$, le dénouement, en mettant fin à la tension narrative, est autant redouté qu'attendu. En interrompant au point maximal de la tension l'épisode de l'attaque des requins, Desnos refuse cette retombée. On peut interpréter de façon parodique, ou du moins ludique, cette exacerbation finale du suspense, qui fait signe vers les procédés du roman-feuilleton :

[...] l'un des marins l'ayant observée s'écria soudain : « Les requins! Les requins !»

C'étaient eux en effet, ils approchaient par rapides coups de queue et, de la digue où tout Nice était groupé, un grand cri d'angoisse s'éleva. Les barques se mirent à fuir, mais les requins n'étaient pas loin. Brusquement, ils plongèrent. Un instant long et tragique puis les flots se colorèrent de rouge. C'était du sang. Puis quelques requins reparurent qui foncèrent sur les barques. C'est alors que le Corsaire Sanglot ${ }^{34} . .$.

Tous les ressorts romanesques sont utilisés pour irriter le désir du lecteur de connaître la suite de l'histoire : la soudaineté de l'attaque, l'angoisse des spectateurs de la scène, l'ignorance dans laquelle le lecteur est maintenu quant à la provenance du sang, l'adverbe «alors» qui annonce une action du héros. Que l'emploi de ces ficelles soit ludique, elles n'en suscitent pas moins ce suspense considéré comme propre à la littérature commerciale, alors que la curiosité, plus intellectuelle, est jugée davantage compatible avec la littérature de création, ce que souligne Raphaël Baroni dans « La valeur littéraire du suspense » où il lie en outre l'efficacité de ce ressort narratif avec l'investissement libidinal qu'il permet ${ }^{35}$.

Dans le roman surréaliste, l'effet privilégié par l'utilisation du stéréotype est pourtant, à première vue, la curiosité. La scène énigmatique à laquelle assiste le narrateur des Dernières Nuits de Paris intrigue et l'explication qu'il en fournit relance cette curiosité : le schéma explicatif que fournit le stéréotype romanesque sera-t-il ou non une description adéquate de la scène? L'utilisation des scènes-types de la littérature romanesque par Aragon dans Anicet ou le panorama, roman produit un effet de curiosité reposant également sur la convocation d'une norme: le lecteur, comme d'ailleurs Anicet lui-même, se demande si le stéréotype va être mené jusqu'à son terme ou si le schéma sera finalement transgressé. La surprise que constitue la fin du roman est amenée par une telle transgression: la présentation de Mirabelle comme une femme fatale et le stéréotype de la femme demandant la grâce de son amant font attendre la libération d'Anicet, qui est pourtant exécuté. L'échec de la démarche est certes également disponible dans l'encyclopédie romanesque - on pense bien sûr ici au Rouge et le Noir: l'effet de transgression est plutôt produit par la combinaison de l'issue malheureuse et d'une mise à distance de la mort du héros, produite par le soudain 
décentrement du roman qui abandonne Anicet au profit d'une focalisation sur Baptiste Ajamais.

Cependant, si seul Desnos, dans notre corpus, thématise le plaisir de la lecture identificatoire favorisée par le roman à suspense, cet investissement libidinal est présent également dans Anicet, où l'on s'interroge par exemple sur l'issue de la démarche de Mirabelle, ou dans À la dérive, où la capture de David Aubry par une bande de voleurs fait craindre pour sa vie: la surveillance constante dont il fait d'abord l'objet, son intégration apparente dans la bande, l'alliance conclue avec un compagnon, la scène finale de l'évasion dont la réussite clôt le chapitre VI ménagent une tension croissante jusqu'à la détente qui ferme l'épisode avant que le chapitre suivant n'annonce un nouveau départ, promettant une nouvelle aventure. Si dans tous ces romans, les ruptures de ton, les effets de montages entre différents genres, les interventions d'auteur et la perturbation de la cohérence d'ensemble du récit favorisent une lecture distanciée, l'utilisation de morceaux intrigués invite en même temps, au niveau local, à une lecture de type identificatoire : le stéréotype, en faisant signe vers la littérature romanesque, renforce l'incitation à cette lecture au premier degré qu'inscrivent dans le texte les ressorts éprouvés du suspense.

Ce déjà-dit ne joue donc pas simplement un rôle critique, mais convoque au sein de la littérature avant-gardiste les plaisirs inavoués de la littérature populaire dont le caractère répétitif accentue encore la dimension libidinale. La gratuité même des péripéties et des reconnaissances - comme, dans Anicet ou le panorama, roman, la présence, sous de multiples déguisements, de Nick Carter dont la justification narrative se fait longtemps attendre - est la marque, dans ces textes, d'un plaisir de l'écriture, écho d'un plaisir de lecture. En soulignant la place tenue par la jouissance dans ces récits, nous procédons au même type de "simplification » que celle qu'Anne Reverseau propose dans la lecture des textes automatiques : elle suggère de prendre au sérieux les images automatiques en tâchant, selon un modèle photographique, de les visualiser ${ }^{36}$. Cependant le montage savant de ces textes invite de surcroît à questionner l'effet visé par cette insertion de saynètes romanesques et par l'utilisation narratologique des péripéties les plus invraisemblables.

«Ressusciter le vent »

L'appel à une lecture identificatoire de scènes hyperboliquement romanesques apparaît en effet comme partie prenante de la stratégie visant à mettre à bas l'attitude réaliste. Dans sa simplicité même, c'est ce que met en lumière Babylone; la banale histoire d'adultère que vit la rousse Cynthia avec son père nourrit un romanesque de pacotille chez l'enfant qui pourtant l'éveille à la liberté en la libérant du quotidien :

Alors, l'écœurent les piètres anecdotes, dont se rassasient la haine familiale. Cynthia, déesse rousse, de vos doigts partent des faisceaux de lumière, mais à leur éclat, s'exagère la tristesse des jours, tous pareils. Ennui, beau fils d'orgueil, une enfance déjà se jure de ne jamais accepter, pour elle-même, la répétition des faits et gestes, le ramassis d'histoires dont vivent ce qu'on appelle les « grandes personnes ${ }^{33}$ ».

Le romanesque permet de "ressusciter le vent ${ }^{38}$ ", même si les intrigues sentimentales qui se nouent autour de l'enfant confinent au grotesque, comme la fuite de la grandmère avec le magistrat Petitdemange. Quelque stéréotypé qu'il soit, le romanesque correspond à un besoin d'aventure, de mystère, comme le souligne le narrateur des Dernières Nuits de Paris, évoquant la bande de Volpe : 
L'ennui s'emparait d'eux et, pour le chasser, ils poursuivaient le mystère et créaient des fantômes.

En les quittant, je les nommais parfois les aventuriers sans aventures ${ }^{39}$. de son enfance ${ }^{40} »:$ «Je suscite sur mes pas des monstres qui guettent; ils ne sont pas encore trop malintentionnés à mon égard et je ne suis pas perdu, puisque que je les crains ${ }^{41}$.» Les invraisemblances empruntées au romanesque populaire constituent un entraînement à la croyance : Vagualame, dans Êtes-vous fous ?, croit aveuglément le roman que prédit la voyante, tandis que le lecteur est amené à accepter autant l'insolite d'un enfant bleu ou d'un rat pesant cinquante kilos que le roman d'espionnage aboutissant à la mort de Myrto-Myrta et à sa résurrection en Yolande, ou la coïncidence qui fait de la voyante sa marraine. Les résumés présentés en tête de chapitre, en concaténant les péripéties, soulignent l'emballement du récit dont la cohésion est assurée par des jeux de mots et des reconnaissances.

L'efficacité, à l'intérieur de la diégèse, de l'hypothèse romanesque incite le lecteur à adopter cette attitude romanesque face au monde. À toutes les étapes des Dernières Nuits de Paris, l'interprétation de la scène initiale par le narrateur est vérifiée et sa justesse n'est même jamais mise en question malgré sa haute improbabilité. Lors de sa deuxième rencontre avec le marin, le narrateur le conduit devant l'Institut sans mettre en doute sa qualité d'assassin. Et bien sûr la suite lui donne raison, puisque le marin avoue. Dans ces romans, il est toujours avantageux de jouer l'invraisemblable contre le vraisemblable et de calculer ses coups en fonction de son bagage livresque plutôt que de sa connaissance du réel tel qu'il est - ou plutôt tel qu'il semble être :

Il se compara d'instinct à Michel Strogoff quand l'Émir de Tatarie, au moment qu'il lui va faire brûler les yeux, offre à cet intéressant personnage un ballet réglé par Madame Stichel, et se prépara du mieux qu'il put à jouir de la solution promise ${ }^{42}$.

Le stéréotype romanesque joue en cela un rôle de libération à l'encontre de l'attitude réaliste: «Le romanesque nous rappelle donc que si tout modèle fictionnel est un modèle pour la réalité [...], cela n'implique nullement qu'il doive être un modèle de la réalité : il peut être un modèle contre la réalité ${ }^{43}$. " L'exacerbation des affects, l'accumulation des épisodes et des péripéties, toutes attendues qu'elles puissent être dans les genres populaires, font dans ces romans l'effet d'un écart et d'une libération de l'écriture vis-à-vis des conventions réalistes. C'est aussi cette liberté de l'écriture narrative que Desnos affirme contre le lien amoureux et dont témoigne l'ouverture finale du récit, qui pourrait être continué indéfiniment. En outre, en utilisant les ressorts du suspense et de la curiosité, les surréalistes accentuent la tension vers l'avenir de leur récit qui précipite la lecture, comme l'automatisme précipite l'écriture, et assoupit de la sorte le sens critique du lecteur étourdi par les rebondissements et les surprises.

30 C'est au contraire de cette capacité à l'adhésion dont David Aubry est complètement dépourvu dans À la dérive: il finit par rechercher au sein de l'Église cette foi qui lui a tant manqué ${ }^{44}$. Cette impossibilité à s'impliquer, à s'identifier ne le quitte que lors de l'épisode démarqué du genre du detective novel, qu'il vit justement comme un spectateur - comme un lecteur - et non comme un acteur : "À cette époque, il s'était vraiment oublié et il revivait plus intensément, plus facilement cette période de sa vie parce que pendant ces quelques journées il était un spectateur et non l'acteur ${ }^{45}$. » Ce que garde Soupault du romanesque, c'est la vitesse narrative, mais en en désamorçant la tension - 
et le plaisir - en faisant vivre ce romanesque par un personnage dont la liberté va de pair avec l'indifférence. Le romanesque, dépouillé de son effet sur le lecteur, de son pathos, n'est plus reconnaissable que par son caractère stéréotypé, le lecteur étant alors tendu entre une lecture identificatoire suggérée par son encyclopédie romanesque et le détachement auquel l'invite le point de vue de David Aubry.

Mais si dans cette indifférence on atteint le maximum de liberté, le principe de libération propre au romanesque paraît tout à fait perdu : ce dont libère le romanesque c'est, en même temps que de l'attitude réaliste, de la fuite du temps et du dégoût de vivre. Le romanesque surgit contre le "vague à l'âme " qui donne son nom à René Crevel dans Êtes-vous fous?, guérit, pour un instant, de l'ennui du sana, fait oublier la mort qui vient, en faisant surgir Yolande, la femme fatale, ressuscitée par l'imposition sur sa peau d'un fakir. Mais la parenthèse se referme, René Crevel reparaît derrière le masque de Vagualame et retrouve le sentiment du temps et de la maladie : «Et puis, à quoi bon les divertissements qui grignotent nos minutes, ces rongeurs (comme feue Yolande, son rat), mais ne peuvent rien contre les heures, dont les griffes ont blessé notre désert de soufre ${ }^{46}$ ? " Ces romans riches en péripéties mettent ainsi également en scène des moments de retombée d'où s'absente le romanesque et où le temps est soudain déstructuré en une errance, qui s'accompagne de l'indifférence du héros: "Une femme, une ville lutent d'indifférence ${ }^{47}$ ", comme l'écrit Crevel à la fin de Babylone. À l'inverse, le retour de Georgette, la "femme-mystère », pour emprunter l'expression de $\mathrm{Crevel}^{48}$, à la fin des Dernières Nuits de Paris redonne in extremis au temps son rythme et à la vie des personnages masculins son intérêt :

Et Georgette entra. Le froid la suivait et le matin.

- C'est vous? fit quelqu'un.

- C'est moi, répondit-elle.

Et elle sourit.

Paris était devant nos yeux.

Nous n'attendions plus personne.

$[\ldots]$

Le jour et la nuit reprenaient leur ronde ${ }^{49}$.

Si le stéréotype romanesque est dans l'écriture surréaliste avant tout un principe de plaisir, il est un plaisir précaire, sans cesse menacé par l'écriture ironique et par l'inquiétude qui baigne l'ensemble de la littérature romanesque des années vingt : alors la « croyance se perd ${ }^{50}$ » et ni l'émerveillement des images, ni les invraisemblances du romanesque ne parviennent à racheter le réel tel qu'il est.

Conclusion

Les stéréotypes romanesques permettent d'écrire des romans surréalistes, en évitant les écueils de la psychologie et de la vraisemblance, et contribuent dans le même temps à élargir les bornes de la croyance, en assoupissant le sens critique du lecteur. Ils sont ainsi dirigés, à travers le réalisme, contre le réel lui-même, ou plutôt contre le réel accrédité par l'attitude réaliste. En appliquant à l'histoire qu'ils vivent des schémas puisés dans la littérature romanesque, les personnages proposent au lecteur une grille d'interprétation du réel où l'hypothèse la plus romanesque est toujours la meilleure. Mais la croyance des surréalistes dans le romanesque n'est pas naïve: elle est indissociable d'une dimension ludique, voire ironique. Cependant, cette croyance est maintenue malgré tout et correspond à une jouissance de ces scènes stéréotypées à laquelle n'est pas étranger un plaisir de la réitération qui s'approche du plaisir procuré par le fantasme érotique. Finalement, ce que montre l'utilisation par les surréalistes des 
stéréotypes romanesques, que la recherche avant-gardiste de la surprise et du nouveau aurait dû battre en brèche, c'est, conformément aux récentes théories de la lecture ${ }^{51}$, la coexistence, dans la littérature de création comme dans la littérature dite commerciale, de deux lectures : la lecture linéaire et identificatoire, et la lecture compréhensive et esthétique. Le caractère stéréotypé des scènes empruntées à la littérature populaire montre en somme leur force passionnante dont les surréalistes captent les pouvoirs pour tâcher de vivre de façon passionnelle ${ }^{52}$.

\section{BIBLIOGRAPHIE}

ARAGON Louis, Anicet ou le panorama, roman [1921], Paris, Gallimard, 2001.

BARONI Raphaël, La tension narrative, Paris, Éditions du Seuil, 2007.

ID., « La valeur littéraire du suspense », A contrario, n. 1, vol. 2, 2004, pp. 29-43.

BERRANGER Marie-Paule, «La liberté ou l'amour ! Récit poétique », in Desnos pour l'an 2000, Actes du colloque de Cerisy (2000), sous la direction de K. Conley et M.-C. Dumas, Paris, Gallimard, 2000, pp. $167-180$.

BLETON Paul, Ça se lit comme un roman policier. Comprendre la lecture sérielle, Éditions Nota Bene, 1999. BOUCHARENC Myriam, L'échec et son double. Philippe Soupault romancier, Paris, Champion, 1997.

BRETON André, Cuvres complètes, édition établie par M. Bonnet, vol. I, Paris, Gallimard, 1988.

CHÉNIEUX-GENDRON Jacqueline, Le surréalisme et le roman, Lausanne, L’Âge d'Homme, 1983.

COMBE Dominique, «L'œil existe à l'état sauvage », Mélusine, n. 21, 2001, pp. 9-24.

CREVEL René, Babylone [1927], Paris, Jean-Jacques Pauvert, 1991.

ID., Ettes-vous fous ? [1929], Paris, Gallimard, 1997.

DESNOS Robert, La liberté ou l'amour ! [1927], Paris, Gallimard, 2001.

DUMAS Marie-Claire, «Surréalisme et stéréotype », in Le stéréotype. Crise et transformations, Actes du colloque de Cerisy-la-Salle (1993), sous la direction d'A. Goulet, Caen, Presses Universitaires de Caen, 1994, pp. 165-171.

GERVAIS Bertrand, À l'écoute de la lecture, Montréal, VLB Éditeur, 1993.

JOUVE Vincent, L'effet-personnage dans le roman, Paris, Presses Universitaires de France, 1992.

REVERSEAU Anne, « Microlectures des textes automatiques surréalistes : complexité, simplicité et complications », Fabula LHT, n. 3, 01/09/2007 (http://www.fabula.org/lht/3/Reverseau.html) RIVIÈRE Jacques, « Le roman d'aventure », La nouvelle revue française, n. 55, juillet 1913, pp. 56-77. SCHAEFFER Jean-Marie, « La catégorie du romanesque », in Le romanesque, sous la direction de G. Declercq et M. Murat, Paris, Presses de la Sorbonne Nouvelle, 2004, pp. 291-302.

SOUPAult Philippe, À la dérive, Paris, J. Ferenczi et fils éditeurs, 1923. 


\section{NOTES}

1. Jacqueline Chénieux-Gendron, Le surréalisme et le roman, Lausanne, L'Âge d'Homme, 1983.

2. Voir, par exemple, Dominique Combe, «L'œil existe à l'état sauvage ", Mélusine, n. 21, 2001, p. 9.

3. André Breton, Manifeste du surréalisme [1924], in ID. Euvres complètes, édition établie par Marguerite Bonnet, vol. I, Paris, Gallimard, 1988, p. 315.

4. Ibid., p. 320.

5. Marie-Claire Dumas, "Surréalisme et stéréotype », in Le stéréotype. Crise et transformations, Actes du colloque de Cerisy-la-Salle (1993), sous la direction d'A. Goulet, Caen, Presses Universitaires de Caen, 1994, pp. 165-171.

6. Voir Myriam Boucharenc, L'échec et son double. Philippe Soupault romancier, Paris, Champion, 1997, p. 230.

7. André Breton, Manifeste du surréalisme, op. cit., p. 332.

8. Louis Aragon, Anicet ou le panorama, roman [1921], Paris, Gallimard, 2001, p. 65.

9. Ibid., p. 84.

10. René Crevel, Êtes-vous fous? [1929], Paris, Gallimard, 1997, p. 20.

11. Ibid., p. 142.

12. Raphaël Baroni en a récemment mis en évidence le rôle dans les narrations intriguées. Voir Raphaël Baroni, La tension narrative, Paris, Éditions du Seuil, 2007.

13. Robert Desnos, La liberté ou l'amour ! [1927], Paris, Gallimard, 2001, p. 21.

14. Ibid., p. 22.

15. Louis Aragon, Anicet ou le panorama, roman, op. cit., p. 115.

16. Ibid., p. 165-166.

17. Ibid., p. 166. Au contraire, l'auteur ne désigne pas à l'attention du lecteur la citation de Fantômas reprenant au sujet d'une petite fille autrefois aimée d'Anicet la phrase caractérisant Lady Beltham : «Je lui trouvais un port de reine. » (ibid., p. 194)

18. André Breton, Manifeste du surréalisme, op. cit., p. 315.

19. Louis Aragon, Anicet ou le panorama, roman, op. cit., p. 197.

20. Robert Desnos, La liberté ou l'amour !, op. cit., p. 52.

21. Philippe Soupault, À la dérive, Paris, J. Ferenczi et fils, éditeurs, 1923, p. 77.

22. André Breton, Manifeste du surréalisme, op. cit., p. 315.

23. René Crevel, Êtes-vous fous ?, op. cit., p. 43.

24. Jacques Rivière, «Le roman d'aventure », La Nouvelle Revue française, n. 55, juillet 1913, pp. 56-57.

25. «[...] l'incertitude introduite par le nœud ne produit l'attente d'un dénouement que dans la mesure où l'indétermination est ressentie comme provisoire, donc accompagnée par la promesse, plus ou moins explicite, d'une résolution. », Raphaël Baroni, La tension narrative, op. cit., p. 227.

26. René Crevel, Êtes-vous fous ?, op. cit., p. 88-89. L'histoire de Cour de goitreuse s'étend ensuite de la page 89 à la page 93.

27. Philippe Soupault, Les dernières nuits de Paris [1928], Paris, Gallimard, 1997, p. 108.

28. Paul Bleton, Ça se lit comme un roman policier. Comprendre la lecture sérielle, Éditions Nota Bene, 1999. 
29. Marie-Paule Berranger, «La Liberté ou l'amour ! Récit poétique », in Desnos pour l'an 2000, Actes du colloque de Cerisy (2000), sous la direction de K. Conley et M.-C. Dumas, Paris, Gallimard, 2000, p. 167.

30. Robert Desnos, La liberté ou l'amour !, op. cit., p. 44.

31. Ibid., p. 28.

32. Ibid., p. 31.

33. « En général, d'ailleurs, le dénouement, qui met fin à l'immersion dans l'espace imaginaire et ludique de la narrativité, est ressenti comme une frustration ou une nostalgie. », Raphaël Baroni, La tension narrative, op. cit., p. 133.

34. Robert Desnos, La liberté ou l'amour !, op. cit., p. 144.

35. Voir Raphaël Baroni, «La valeur littéraire du suspense ", A contrario, n. 1, vol. 2, 2004, pp. 29-43.

36. Anne Reverseau, « Microlectures des textes automatiques surréalistes : complexité, simplicité et complications ", Fabula LHT, n. 3, 01/09/2007 (http://www.fabula.org/lht/ 3/Reverseau.html)

37. René Crevel, Babylone [1927], Paris, Jean-Jacques Pauvert, 1991, p. 26.

38. Ibid., p. 29.

39. Philippe Soupault, Les dernières nuits de Paris, op. cit., p. 110.

40. André Breton, Manifeste du surréalisme, op. cit., p. 340.

41. Ibid.

42. Louis Aragon, Anicet ou le panorama, roman, op. cit., p. 70 et 71-72.

43. Jean-Marie Schaeffer, "La catégorie du romanesque ", in Le romanesque, sous la direction de G. Declercq et M. Murat, Paris, Presses de la Sorbonne Nouvelle, 2004, p. 301.

44. « Il errait, cherchant un point d'appui, une perche. Tout était détruit par son absence de foi. ", Philippe Soupault, À la dérive, op. cit., p. 201.

45. Ibid., p. 104.

46. René Crevel, Êtes-vous fous ?, op. cit., p. 175.

47. René Crevel, Babylone, op. cit., p. 184.

48. "Et Yolande, c'est la femme-mystère. ", René Crevel, Êtes-vous fous ?, op. cit., p. 48.

49. Philippe Soupault, Les dernières nuits de Paris, op. cit., p. 141.

50. André Breton, Manifeste du surréalisme, op. cit., p. 311.

51. On peut renvoyer ici à la distinction faite par Bertrand Gervais entre économie de la progression et économie de la compréhension, dont il souligne la complémentarité, ou à celle, rappelée par Vincent Jouve, entre playing et game. Vincent Jouve, pour sa part, distingue, en réaménageant les travaux de Michel Picard, le lectant (activité distanciée), le lu (l'investissement fantasmatique absolu) et le lisant (l'investissement affectif). Voir Bertrand Gervais, À l'écoute de la lecture, Montréal, VLB Éditeur, 1993 et Vincent Jouve, L'effet-personnage dans le roman, Paris, Presses Universitaires de France, 1992, pp. 81-82 notamment.

52. Nous jouons ici sur le couple forgé par Raphaël Baroni : « Dans l'espace du récit, les leçons que nous tirons habituellement des épreuves que nous réserve l'existence peuvent être enseignées sans danger : le vécu passionnel se convertir en histoire passionnante. ", Raphaël Baroni, La tension narrative, op. cit., p. 35. 


\section{RÉSUMÉS}

L'utilisation du stéréotype romanesque par les surréalistes dans les années vingt permet d'écrire des romans surréalistes évitant les ornières du roman réaliste dénoncées par André Breton : les "grosses ficelles» du roman populaire fonctionnent comme un «secret de l'art magique surréaliste » en nouant la narration et en évitant l'écueil de la psychologie. Mais à cet usage narratologique et critique se combine une jouissance de la lecture et de l'écriture romanesques qui apparie le stéréotype au fantasme érotique. En jouant, de surcroit, des invraisemblances romanesques, les surréalistes battent en brèche l'attitude réaliste face au monde et nourrissent le sens du merveilleux nécessaire à l'émergence du surréel. L'emploi ludique du stéréotype s'accompagne ainsi de l'exploitation de ses ressources passionnantes.

The use of novelistic stereotypes by surrealists during the 1920s permitted the composition of literary works while avoiding the problem of the realistic novel genre that André Breton denounced; the obvious tricks of the popular novel work as "a secret of surrealist art" by structuring narration and avoiding the trap of psychology. However, this narratological and critical use is combined with the pleasure of novel reading and writing that links stereotypes to erotic fantasies. By playing, moreover, on novelistic improbabilities, surrealists demolish the realistic attitude towards the world and nourish the sense of fantasy that is necessary to the emergence of the surreal. Along with a playful use of stereotypes, surrealists fully exploit their fascinating possibilities.

\section{INDEX}

Mots-clés : roman, Soupault Philippe, Desnos Robert, Aragon Louis, populaire, romanesque, surréalisme, stéréotype, suspense, Crevel René

Keywords : narration

\section{AUTEUR}

\section{IVANNE RIALLAND}

PRAG à l'IUT de Marne-la-Vallée - Équipe "Littératures françaises du XXe siècle" (EA 2577), Paris-Sorbonne 